\title{
The Exponentiated Kumaraswamy-Weibull Distribution with Application to Real Data
}

\author{
Fathy Helmy Eissa ${ }^{1}$ \\ ${ }^{1}$ College of Science and Arts- Rabigh, King Abdulaziz University, Saudi Arabia \\ Department of Mathematics, Faculty of Science, Damanhour University, Egypt \\ Correspondence: Fathy H. Eissa, College of Science and Arts- Rabigh, King Abdulaziz University, Saudi Arabia. \\ E-mail: fkhamis@kau.edu.sa, fathy_eissa@hotmail.com.
}

Received: September 16, 2017 Accepted: October 4, 2017 Online Published: October 30, 2017

doi:10.5539/ijsp.v6n6p167 URL: https://doi.org/10.5539/ijsp.v6n6p167

\begin{abstract}
A new five-parameter lifetime distribution called the exponentiated Kumaraswamy-Weibull distribution is introduced. It includes several important sub-models as special cases such as exponentiated Weibull, Kumaraswamy-Weibull, exponentiated exponential, exponentiated Rayleigh and Weibull. Essential mathematical and statistical properties for the distribution are presented. An approximate form of the mode is derived and it can be used to derive mode forms of other well-known distributions. Important parametric characterizations for probability density and hazard functions are discussed. The estimation of the parameters by maximum likelihood method is discussed. Three real data sets are used to show its excellent performance fit over existing popular lifetime models. It is effective model to analyze several positive data sets.
\end{abstract}

Keywords: Asymptotes, Order statistics, Mode, Hazard function, Kumaraswamy-Weibull, moments, maximum likelihood method

\section{Introduction and Motivation}

Kumaraswamy (1980) introduced a two-parameter distribution, defined on $(0,1)$, known as Kumaraswamy distribution and its cumulative distribution function (cdf) is defined for a random variable $\mathrm{X}$, as $F(x)=1-\left(1-x^{a}\right)^{b}, 0<x<1, a>0$ and $b>0$ are shape parameters. Jones(2009) discussed important background and several advantages of this distribution. For an arbitrary baseline cdf $\mathrm{G}(\mathrm{x})$ of a random variable X, Cordeiro and Castro (2011) defined a new family of Kumaraswamy generalized (K-G) distributions with cdf $F(x)=1-\left[1-G^{a}(x)\right]^{b}$. Several widely known distributions have been extended based on K-G distributions by many authors. Paranaiba et al.(2012) introduced the K-BurrXII distribution, Cordeiro et al. (2010) introduced the K-Weibull (K-W) distribution, Pascoa et al. (2011) proposed the K-generalized gamma distribution, Gomes et al. (2014) studied the K-generalized Rayleigh (K-Ray) distribution, among others. For an arbitrary baseline cdf, $\mathrm{G}(\mathrm{x})$, of a random variable, $\mathrm{X}$, the cdf of a generalized class of distributions is defined by

$$
F(x)=\left[1-\left(1-G^{a}(x)\right)^{b}\right]^{\theta}
$$

and it is called the exponentiated Kumaraswamy generalized (EK-G) family. Huang and Oluyede (2014) have used the $\mathrm{G}(\mathrm{x})$ as the cdf of Dagum distribution to define the exponentiated Kumaraswamy-Dagum distribution. The exponentiated Kumaraswamy-exponential (EK-E) distribution was introduced by Rodrigues and Silva (2015). In this article, we propose and study a new generalization for Kumaraswamy-Weibull distribution, named the exponentiated Kumaraswamy-Weibull (EK-W) distribution. We provide a description of its essential characterizations with the hope that it will be useful in applications in reliability and other fields of research. The cdf of the EK-W distribution is obtained by combining $G(x)=1-e^{-(\lambda x)^{\beta}}$ the cdf of the Weibull distribution, and equation (1). This gives the cdf of EK-W distribution as

$$
F(x)=\left\{1-\left[1-\left(1-e^{-(\lambda x)^{\beta}}\right)^{a}\right]^{b}\right\}^{\theta}
$$

and the corresponding probability densty function (pdf) is

$$
\begin{gathered}
f(x)=\lambda \beta a b \theta(\lambda x)^{\beta-1} e^{-(\lambda x)^{\beta}}\left(1-e^{-(\lambda x)^{\beta}}\right)^{a-1}\left[1-\left(1-e^{-(\lambda x)^{\beta}}\right)^{a}\right]^{b-1} \times \\
\\
\left\{1-\left[1-\left(1-e^{-(\lambda x)^{\beta}}\right)^{a}\right]^{b}\right\}^{\theta-1}
\end{gathered}
$$

where $x>0, \lambda$ is the scale parameter and $\beta, a, b$ and $\theta$ are shape parameters of positive real values. A random variable $X$ following equation (3) is denoted by $X \sim E K-W(x ; \lambda, \beta, a, b, \theta)$. For two different groups of the parameters values, 

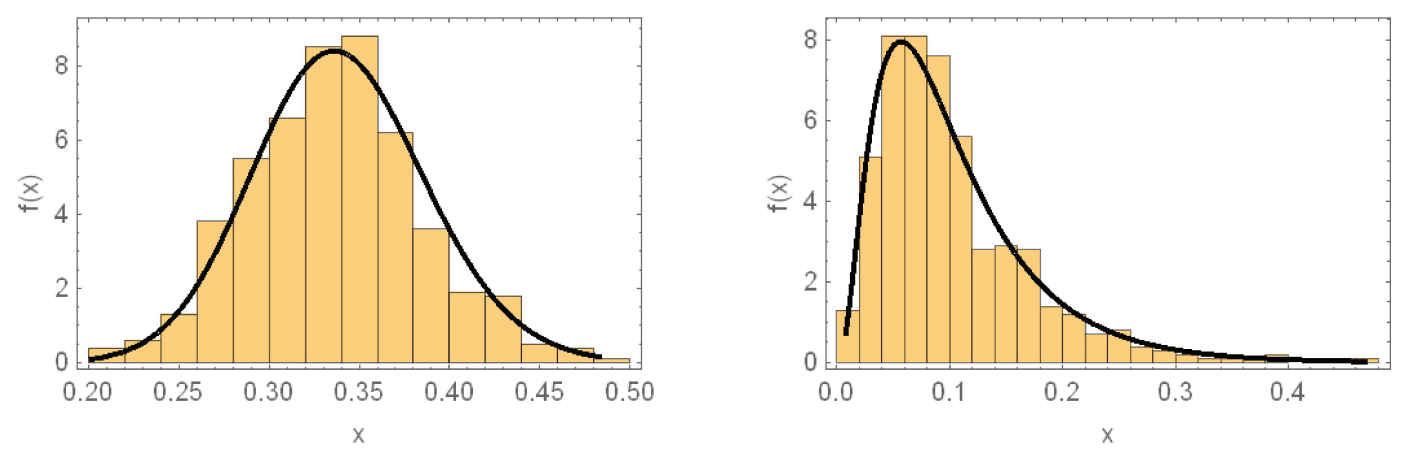

Figure 1. The estimated pdf of EK-W for simulated data sets.

plots of the fitted EK-W density functions and the histograms of two simulated data sets are displayed in Figure 1. The motivations for introducing the EK-W distribution are the following: (i) It is a generalization of the most important wellknown distributions such as Kumaraswamy Wiebull, exponentiated Weibull (EW), Wiebull and exponentiated exponential distributions and it is much more flexible than these distribu tions, as we shall see later in this context. (ii) It could be an important model in a variety of problems in reliability analysis, since its hazard rate function allows for all hazard shapes: monotonically increasing and decreasing, bathtub and unimodal hazard rates, as we shall see later. (iii) The EK$\mathrm{W}$ distribution has physical interpretation in the field of the reliability of systems engineering. Specifically, for $\theta=1$, we have $R(x)=R_{E W}^{b}(x)$ where $R_{E W}(x)$ is the reliability function of the EW distribution. Thus, the reliability of a series system containing an integer number $b$ components whose independent lifetimes follow $E W(\lambda, \beta, a)$ distribution, can be modeled as EK-W distribution. For $b=1$, we have $R(x)=1-\left[1-R_{E W}(x)\right]^{\theta}$ or $R(x)=1-\left[1-R_{w}(x)\right]^{a \theta}, R_{W}(x)$ is the reliability function of the Weibull distribution. Thus, the reliability of a parallel system containing an integer number $\theta(a \theta)$ components whose independent lifetimes follow $E W(\lambda, \beta, a)(W(\lambda, \beta))$ can be modeled as the EK-W distribution. For $a=1$, we have $R(x)=R_{E W}(x)$ or $R(x)=1-\left[1-R_{w}(x)\right]^{\theta}$. Thus, the reliability of a component whose lifetime distributed as EK-W has the same reliability as EW distribution or the reliability of a parallel system of an integer number $\theta$ components whose independent lifetimes follow Weibull distribution. Sub-models of the EK-W distribution are in the following:

- When $\theta=1$ the EK-W reduces to $K-W(\lambda, \beta, a, b)$,

- When $a=b=1$ the EK-W reduces to $E W(\lambda, \beta, \theta)$,

- When $b=1$ the EK-W reduces to $E W(\lambda, \beta, a \theta)$,

- When $\beta=1$ the EK-W reduces to $E K-E(\lambda, a, b, \theta)$,

- When $\beta=b=1$ the EK-W reduces to $E E(\lambda, a \theta)$,

- When $\beta=2, b=\theta=1$ the EK-W reduces to $\operatorname{ERay}(\lambda, a)$,

- When $a=b=\theta=1$ the EK-W reduces to $W(\lambda, \beta)$,

- When $\beta=a=b=\theta=1$ the EK-W reduces to $E(\lambda)$.

The rest of the paper is organized as follows. In Section 3, other representations of the cdf and pdf are derived. Mathematical properties of the EK-W distribution including quantile and simulation, the shape of the pdf, moments and moment generating function, mean deviations, Bonferoni and Lorenz curves, Rényi entropy, order statistics are considered through Section 3 to Section 9. In addition, we study the asymptotes of the hazard rate function, $h(x)$, as $x$ approaches zero and infinity, and its parametric characterizations are discussed in Section 10. We present the estimation of the parameters by maximum likelihood method in Section 11. Applications to three real data sets are carried out in Section 12. Conclusions are given in Section 13.

\section{Expansion for the CDF and PDF}

Other representations of the cdf and pdf of the EK-W distribution can be helpful in numerical applications. Expanding the term $\left\{1-\left[1-\left(1-e^{-(\lambda x)^{\beta}}\right)^{a}\right]^{b}\right\}^{\theta-1}$ in equation (3), sequentially, using the binomial theorem, the cdf $F(x)$ and pdf $f(x)$ of the 
EK-W distribution can be written as alinear combination of the cdf, $F_{W}(x)$, and pdf, $f_{W}(x)$, of the well-known Weibull distribution. For real and non-integers $a>0, b>0$ and $\theta>0$, the forms of $F(x)$ and $f(x)$ are, respectively, given by

$$
F(x)=\sum_{i, j, k=0}^{\infty} S(i, j, k) F_{W}\left(x ; \lambda_{k}, \beta\right)
$$

and

$$
f(x)=\sum_{i, j, k=0}^{\infty} S(i, j, k) f_{W}\left(x ; \lambda_{k}, \beta\right)
$$

where

$$
\lambda_{k}=\lambda(k+1)^{\frac{1}{\beta}}
$$

and

$$
S(i, j, k)=\frac{a b \theta(-1)^{i+j+k} \Gamma(\theta) \Gamma[b(i+1)] \Gamma[a(j+1)]}{i ! j ! k ! \Gamma(\theta-i) \Gamma[b(i+1)-j] \Gamma[a(j+1)-k](k+1)} .
$$

The sum of the weights $S(i, j, k)$ is equal to one via Mathematica 10.0, say. The form of $f(x)$ in equation (5) can be helpful to obtain some mathematical properties of the EK-W distribution directly from these of the conventional Weibull distribution such as the moments, moment generating function and characteristic function.

\section{Quantile Function and Simulation Variable}

The p-th quantile, $x_{p}$, of the EK-W distribution is the solution of the equation $F\left(x_{p}\right)=p, 0<p<1$ and it is given by

$$
x_{p}=\frac{1}{\lambda}\left\{-\log \left[1-\left[1-\left(1-p^{1 / \theta}\right)^{1 / b}\right]^{1 / a}\right]\right\}^{1 / \beta} .
$$

For $p=0.5$, the median, $x_{m}$, of $f(x)$ is

$$
x_{m}=\frac{1}{\lambda}\left\{-\log \left[1-\left[1-\left(1-2^{-1 / \theta}\right)^{1 / b}\right]^{1 / a}\right]\right\}^{1 / \beta} .
$$

Simulating EK-W random variable is easily obtained by considering $X=F^{-1}(u)$. Thus, one can generate EK-W variates by

$$
X=\frac{1}{\lambda}\left\{-\log \left[1-\left[1-\left(1-U^{1 / \theta}\right)^{1 / b}\right]^{1 / a}\right]\right\}^{1 / \beta} .
$$

where $U$ is a uniformly distributed random variable on $(0,1)$ interval.

\section{Shape of the PDF}

The mode is an important key to identify the shape of the pdf $f(x)$ given by equation (3). We provide the following theorem for the shape of the density function $f(x)$.

Theorem 1 The pdf of the EK-W distribution is unimodal and has a unique mode at $x=x_{d}$ when $\beta \theta a>1$ and the approximate form of the mode is given by

$$
x_{d} \approx \begin{cases}\frac{1}{\lambda}\left[\frac{2(\beta \theta a-1)}{\beta b(\theta a+1)}\right]^{\frac{1}{\beta}} & \text { when } \quad \beta \theta a>1 \\ 0 & \text { when } \quad \beta \theta a \leq 1 .\end{cases}
$$

Proof. The mode is the value corresponding to the point at which the slope of the tangent of the curve of $f(x)$ is zero. Differentiating $f(x)$ wih respect to $x$, we get

$$
\begin{gathered}
f^{\prime}(x)=g(x) f(x), \\
g(x)=\lambda(\beta-1)(\lambda x)^{-1}-\lambda \beta(\lambda x)^{\beta-1}+\lambda \beta(a-1)(\lambda x)^{\beta-1} u^{-1}(1-u) \\
+\lambda \beta a b(\theta-1)(\lambda x)^{\beta-1} u^{a-1}(1-u)\left(1-u^{a}\right)^{b-1}\left[1-\left(1-u^{a}\right)^{b}\right]^{-1} \\
-\lambda \beta a(b-1)(\lambda x)^{\beta-1} u^{a-1}(1-u)\left(1-u^{a}\right)^{-1} .
\end{gathered}
$$




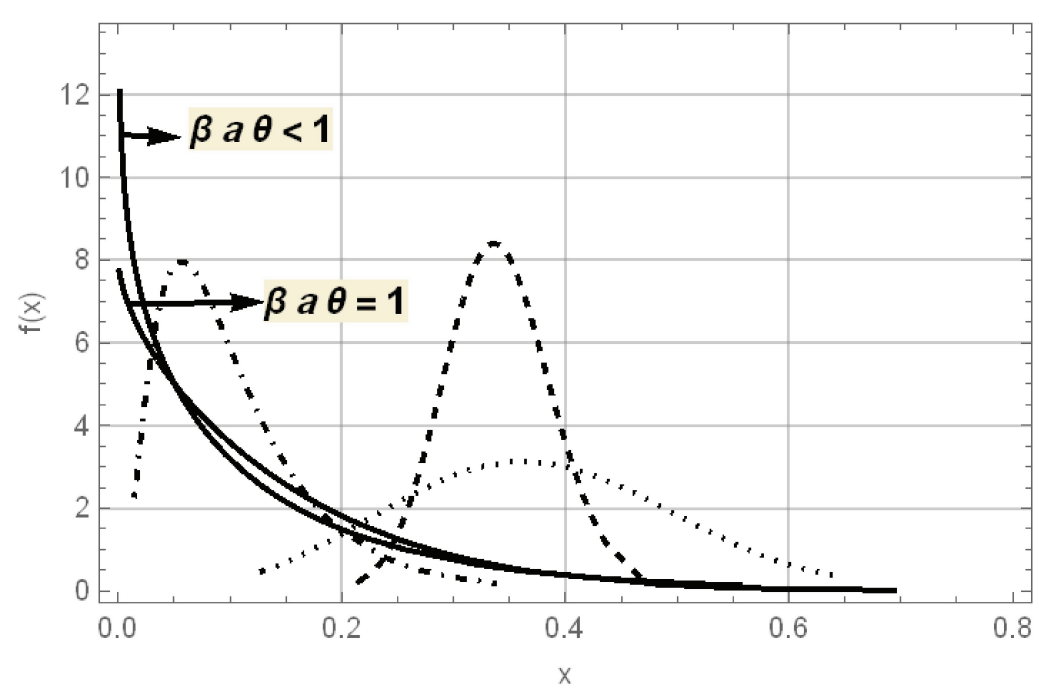

Figure 2. The pdfs of EK-W for various parameter values.

where $u=u(x)=1-e^{-(\lambda x)^{\beta}}$. At $f^{\prime}(x)=0$, we have $f(x) \neq 0$ and it must be $g(x)=0$. Therefore, the mode of $f(x)$ is at the value of $x=x_{d}$ satisfying $g(x)=0$. Consequently, we have

$$
\begin{aligned}
& \beta-1-\beta(\lambda x)^{\beta}+\beta(a-1)(\lambda x)^{\beta} u^{-1}(1-u) \\
& +\beta a b(\theta-1)(\lambda x)^{\beta} u^{a-1}(1-u)\left(1-u^{a}\right)^{b-1}\left[1-\left(1-u^{a}\right)^{b}\right]^{-1} \\
& -\beta a(b-1)(\lambda x)^{\beta} u^{a-1}(1-u)\left(1-u^{a}\right)^{-1}=0 .
\end{aligned}
$$

For positive real values of the parameters $\lambda, \beta, a, b$ and $\theta$, one can obtain an exact value of the mode by solving equation (10), numerically. On the other hand, after some algebra and approximations for some terms of equation (10), we can obtain an approximate explicit form of the mode as given in equation (9). This completes the proof.

This form of the mode is an explicit form in terms of the parameters and it detects information about the shape of the pdf $f(x)$ of the EK-W distribution in terms of certain combination of the parameters values. The density function $f(x)$ of the EK-W distribution exhibits two different behaviors. The curve of $f(x)$ is unimodal when $\beta a \theta>1$ and it is decreasing when $\beta a \theta \leq 1$. For selected parameter values, plots of $f(x)$ are displayed in Figure 2 in the cases of $\beta a \theta \leq 1$ and $\beta a \theta>1$. The form of the mode in equation (9) gives explicit formulas of the mode of well-known distributions that have no known mode forms as special cases. Mathematical formulas of the modes for some sub-models are obtained and presented in Table 1.

Furthermore, the asymptotes of $f(x)$ can be obtained as

$$
f(x) \approx \lambda \beta a \theta b^{\theta}(\lambda x)^{\beta a \theta-1} \text {, as } \quad x \rightarrow 0
$$

and

$$
f(x) \approx \lambda \beta b \theta a^{b}(\lambda x)^{\beta-1} e^{-b(\lambda x)^{\beta}} \text {, as } x \rightarrow \infty
$$

In terms of the left and right tails of $f(x)$ curve, we introduce the following theorem.

Theorem 2 The limit of $E K-W(\lambda, \beta, a, b, \theta)$ density function $f(x)$ is zero as $x \rightarrow \infty$ and its limit as $x \rightarrow 0$ is given by

$$
\lim _{x \rightarrow 0} f(x)= \begin{cases}0 & \text { when } \quad \beta a \theta>1 \\ \lambda b^{\theta} & \text { when } \quad \beta a \theta=1 \\ \infty & \text { when } \quad \beta a \theta<1\end{cases}
$$

Proof. From the results obtained in equations (11) and (12), the proof is straightforward to set. 
Table 1. Mode forms for some distributions.

\begin{tabular}{l|l}
\hline Distribution & Mode Form \\
\hline$K-W(\lambda, \beta, a, b)$ & $\frac{1}{\lambda}\left[\frac{2(\beta a-1)}{\beta b(a+1)}\right]^{1 / \beta}, \quad \beta a>1$ \\
$E W(\lambda, \beta, \theta)$ & $\frac{1}{\lambda}\left[\frac{2(\beta \theta-1)}{\beta(\theta+1)}\right]^{1 / \beta}, \quad \beta \theta>1$ \\
$E W(\lambda, \beta, a)$ & $\frac{1}{\lambda}\left[\frac{2(\beta a-1)}{\beta(a+1)}\right]^{1 / \beta}, \quad \beta a>1$ \\
$E K-E(\lambda, a, b, \theta)$ & $\frac{1}{\lambda}\left[\frac{2(a \theta-1)}{b(a \theta+1)}\right], \quad a \theta>1$ \\
$K-E(\lambda, a, b)$ & $\frac{1}{\lambda}\left[\frac{2(a-1)}{b(a+1)}\right], \quad a>1$ \\
$E E(\lambda, a \theta)$ & $\frac{1}{\lambda}\left[\frac{2(a \theta-1)}{a \theta+1}\right], \quad a \theta>1$ \\
$K-R a y(\lambda, a, b)$ & $\frac{1}{\lambda}\left[\frac{2 a-1}{b(a+1)}\right]^{1 / 2}, \quad a>1 / 2$ \\
$W(\lambda, \beta)$ & $\frac{1}{\lambda}\left[\frac{\beta-1)}{\beta}\right]^{1 / \beta}, \quad \beta>1$ \\
\end{tabular}

The results of Theorem 2 are shown and supported by Figure 2. Figure 2 displays some curves of pdf when $\beta a \theta>1$ and $\beta a \theta \leq 1$. For example, for the case of $\beta a \theta=1$, we assumed $\beta=2, a=.333, \theta=1.5, \lambda=2$ and $b=2.5$, Figure 2 shows that $f(0)=7.9$.

\section{Moments and Moment Generating Function}

The r-th ordering moment of the EK-W distribution can be formed as infinite (finite) weighted linear combination of those quantities for the well-known Weibull distribution. Other moments such as central and factorial moments can be calculated too. We have the r-th moment of the $W(\lambda, \beta)$ to be $\mu_{r}=\lambda^{-r} \Gamma\left(\frac{r}{\beta}+1\right)$. Therefore, the r-th moment of EK-W random variable $X$ can be obtained as

$$
\mu_{r}=\sum_{i, j, k=0}^{\infty} S(i, j, k)\left(\lambda_{k}\right)^{-r} \Gamma\left(\frac{r}{\beta}+1\right)
$$

where $\lambda_{k}$ and the weights $S(i, j, k)$ are defined in equation (4). The summation symbols are abbreviated to one with three indecises. Equation (14) can be rewritten in the form

$$
\begin{aligned}
\mu_{r}= & a b \theta \Gamma\left(\frac{r}{\beta}+1\right) \times \\
& \sum_{i, j, k=0}^{\infty} \frac{(-1)^{i+j+k} \Gamma(\theta) \Gamma[b(i+1)] \Gamma[a(j+1)]}{i ! j ! k ! \Gamma(\theta-i) \Gamma[b(i+1)-j] \Gamma[a(j+1)-k](k+1) \lambda_{k}^{r}} .
\end{aligned}
$$

The indices $i, j$ and $k$ stop at $(\theta-1), b(i+1)-1$, and $a(j+1)-1$, respectively, when $\theta, b$ and $a$ are integers. In particular, the mean of the EK-W distribution is given by

$$
\begin{aligned}
\mu=E(x)= & a b \theta \Gamma\left(\frac{1}{\beta}+1\right) \times \\
& \sum_{i, j, k=0}^{\infty} \frac{(-1)^{i+j+k} \Gamma(\theta) \Gamma[b(i+1)] \Gamma[a(j+1)]}{i ! j ! k ! \Gamma(\theta-i) \Gamma[b(i+1)-j] \Gamma[a(j+1)-k](k+1) \lambda_{k}} .
\end{aligned}
$$

From the first four ordinary moments obtained by using equation (14), one can calculate the measures of skewness, $\gamma_{1}$, and kurtosis, $\gamma_{2}$, using the well-known relationships:

$$
\gamma_{1}=\frac{\mu_{3}-3 \mu_{1} \mu_{2}+2 \mu_{1}^{3}}{\left[\mu_{2}-\mu_{1}^{2}\right]^{3 / 2}} \quad \text { and } \quad \gamma_{2}=\frac{\mu_{4}-4 \mu_{1} \mu_{3}+6 \mu_{1}^{2} \mu_{2}-3 \mu_{1}^{4}}{\left[\mu_{2}-\mu_{1}^{2}\right]^{2}}
$$

where $\mu_{1}, \mu_{2}, \mu_{3}$, and $\mu_{4}$ can be calculated from equation (14). Based on the quantile functions, we can also compute the skewness, as defined by Galton (1983), and kurtosis, as defined by Moors (1988), as

$$
\gamma_{1}=\frac{Q(6 / 8)-2 Q(4 / 8)+Q(2 / 8)}{Q(6 / 8)-Q(2 / 8)}
$$


and

$$
\gamma_{2}=\frac{Q(7 / 8)-Q(5 / 8)+Q(3 / 8)-Q(1 / 8)}{Q(6 / 8)-Q(2 / 8)} .
$$

where $Q(p)=x_{p}$ is computed from equation (6). Figure 3 and Figure 4 display plots of the skewness $\left(\gamma_{1}\right)$ and kurtosis $\left(\gamma_{2}\right)$, as functions of $\theta$. The plots are carried out for values of $\lambda=2, \beta=2.5$ and selected values for $a$ and $b$.

The moment generating function (mgf), $m(t)$, is defined by
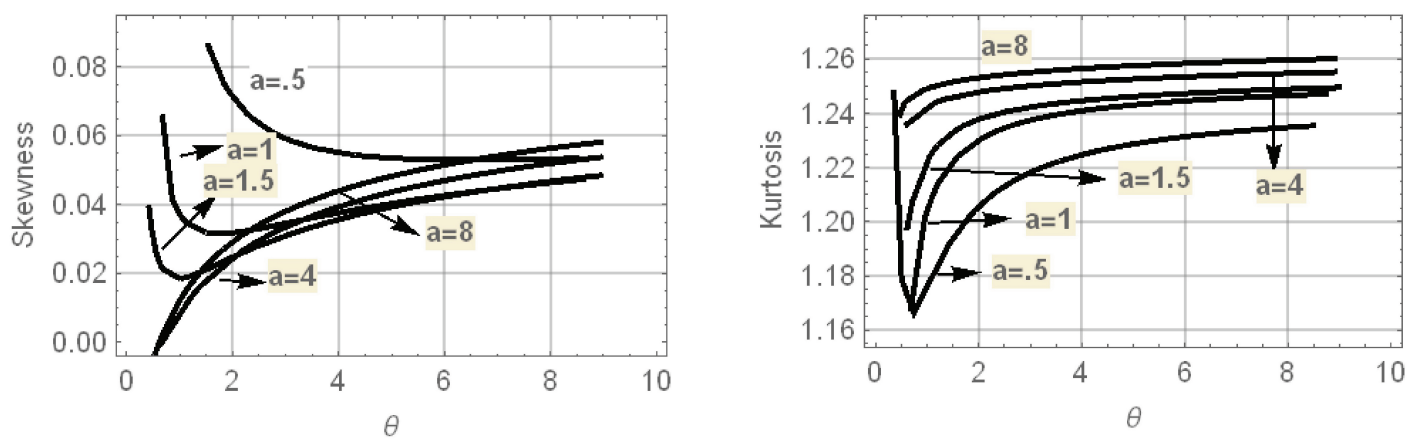

Figure 3. $\gamma_{1}$ and $\gamma_{2}$ as functions of $\theta$ for selected values of $a$.
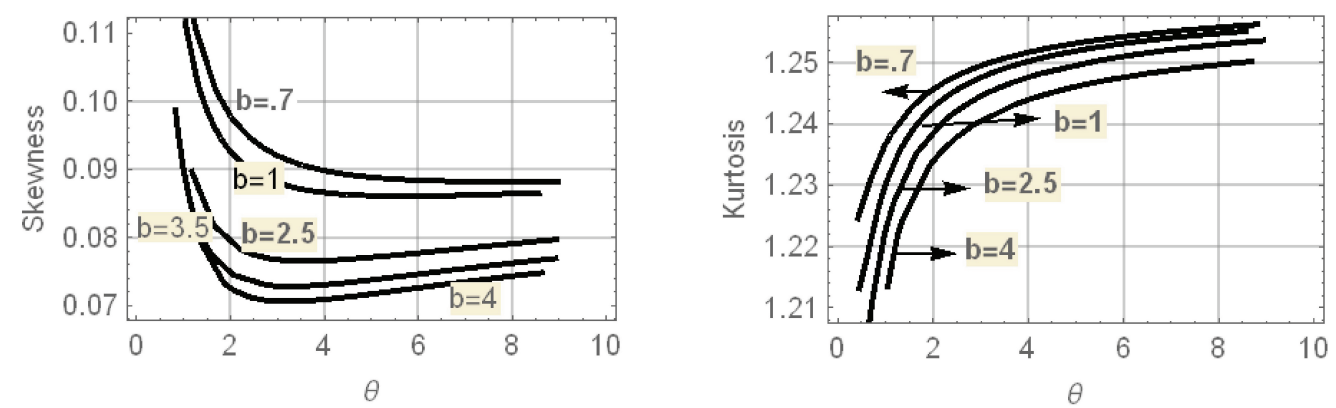

Figure 4. $\gamma_{1}$ and $\gamma_{2}$ as functions of $\theta$ for selected values of $b$.

$$
m(t)=E\left(e^{t X}\right)=\int_{-\infty}^{\infty} e^{t x} f(x) d x
$$

For a random variable $X \sim E K-W$ distribution with pdf $f(x)$ given by equation (3), we get the mgf as

$$
m(t)=\sum_{i, j, k=0}^{\infty} S(i, j, k) \lambda_{k}^{-r} \Gamma\left(\frac{r}{\beta}+1\right) \frac{t^{r}}{r !}, \quad t>0 .
$$

that can be rewritten in the form

$$
\begin{aligned}
m(t)= & a b \theta \times \\
& \sum_{i, j, k, r=0}^{\infty} \frac{(-1)^{i+j+k} \Gamma(\theta) \Gamma[b(i+1)] \Gamma[a(j+1)] t^{r}}{i ! j ! k ! r ! \Gamma(\theta-i) \Gamma[b(i+1)-j] \Gamma[a(j+1)-k](k+1) \lambda_{k}^{r}} \Gamma\left(\frac{r}{\beta}+1\right)
\end{aligned}
$$

for $t>0$.

\section{Mean Deviations}

The amount of spread in a population is measured to some extent by the totality of deviation from the mean (median) which is known as the mean deviation about the mean (median). For $X \sim E K-W(\lambda, \beta, a, b, \theta)$ with mean $\mu$ and median $m$, the mean deviations about the mean and median are respectively, defined by

$$
\delta_{1}(X)=E(|X-\mu|) \quad \text { and } \quad \delta_{2}(X)=E(|X-m|)
$$


where $\mu$ and $m$ are given by equations (16) and (7). From the definition of the expectation, we can obtain $\delta_{1}(X)$ in the form

$$
\delta_{1}(X)=2 \mu \int_{0}^{\mu} f(x) d x-2 \int_{0}^{\mu} x f(x) d x .
$$

Substituting for $f(x)$ by the form in equation (3), we get

$$
\begin{aligned}
\delta_{1}(X) & =2 \mu F(\mu)-2 a b \theta \times \\
& \sum_{i, j, k=0}^{\infty} \frac{(-1)^{i+j+k} \Gamma(\theta) \Gamma[b(i+1)] \Gamma[a(j+1)] \quad \gamma\left(\beta^{-1}+1,\left(\lambda_{k} \mu\right)^{\beta}\right)}{i ! j ! k ! \Gamma(\theta-i) \Gamma[b(i+1)-j] \Gamma[a(j+1)-k](k+1) \lambda_{k}} .
\end{aligned}
$$

and in similar way, we get

$$
\begin{aligned}
\delta_{2}(X) & =\mu-2 a b \theta \times \\
& \sum_{i, j, k=0}^{\infty} \frac{(-1)^{i+j+k} \Gamma(\theta) \Gamma[b(i+1)] \Gamma[a(j+1)] \quad \gamma\left(\beta^{-1}+1,\left(\lambda_{k} m\right)^{\beta}\right)}{i ! j ! k ! \Gamma(\theta-i) \Gamma[b(i+1)-j] \Gamma[a(j+1)-k](k+1) \lambda_{k}} .
\end{aligned}
$$

where $\gamma(a, z)=\int_{0}^{z} u^{a-1} e^{-u} d u, z>0$, is the incomplete gamma function and $\lambda_{k}=\lambda(k+1)^{1 / \beta}$.

\section{Bonferroni and Lorenz Curves}

It is well-known that the Bonferoni and Lorenz curves have applications in several fields such as economic, reliability, medicine and insurance. The Bonferroni and Lorenz curves are respectively, defined by

$$
B(p)=\frac{1}{p \mu} \int_{0}^{q} x f(x) d x \text { and } L(p)=\frac{1}{\mu} \int_{0}^{q} x f(x) d x
$$

where $q=F^{-1}(p)$ and $F($.$) is the distribution function.$

For $X \sim E K-W(\lambda, \beta, a, b, \theta)$, we get

$$
\begin{aligned}
B(p)= & \frac{a b \theta}{p \mu} \times \\
& \sum_{i, j, k=0}^{\infty} \frac{(-1)^{i+j+k} \Gamma(\theta) \Gamma[b(i+1)] \Gamma[a(j+1)]}{i ! j ! k ! \Gamma(\theta-i) \Gamma[b(i+1)-j] \Gamma[a(j+1)-k](k+1) \lambda_{k}}
\end{aligned}
$$

and

$$
\begin{aligned}
L(p)= & \frac{a b \theta}{\mu} \times \\
& \sum_{i, j, k=0}^{\infty} \frac{(-1)^{i+j+k} \Gamma(\theta) \Gamma[b(i+1)] \Gamma[a(j+1)] \quad \gamma\left(\beta^{-1}+1,\left(\lambda_{k} q\right)^{\beta}\right)}{i ! j ! k ! \Gamma(\theta-i) \Gamma[b(i+1)-j] \Gamma[a(j+1)-k](k+1) \lambda_{k}} .
\end{aligned}
$$

where $\mu$ is the mean of $X$, given by equation (16), $\gamma(a, z)$ is the incomplete gamma function and

$$
q=F^{-1}(p)=\frac{1}{\lambda}\left\{-\log \left[1-\left[1-\left(1-p^{1 / \theta}\right)^{1 / b}\right]^{1 / a}\right]\right\}^{1 / \beta}
$$

\section{Rényi Entropy}

An entropy of a random variable $X$ with pdf $f(x)$, is a measure of variation of the uncertainty. It is a concept encountered in engineering, physics and other fields such as theory of communication and probability. Rényi entropy is defined by

$$
E_{R}(v)=\frac{1}{1-v} \log \left[\int f^{v}(x) d x\right]
$$

where $v>0$ and $v \neq 1$. Combining equation (3) and equation (22), Rényi entropy of the $E K-W(\lambda, \beta, a, b, \theta)$ is given by

$$
E_{R}(v)=\frac{v}{1-v} \log (a b \theta)-\log (\lambda \beta)+\frac{1}{1-v} \log (\varphi(v))
$$


where

$$
\varphi(v)=\sum_{i, j, k=0}^{\infty} \frac{(-1)^{i+j+k}}{(k+v)^{[v(\beta-1)] / \beta}}\left(\begin{array}{c}
v(\theta-1) \\
i
\end{array}\right)\left(\begin{array}{c}
v(b-1+b i) \\
j
\end{array}\right)\left(\begin{array}{c}
v(a-1)+a j \\
k
\end{array}\right) .
$$

\section{Order Statistics}

Order statistics play a prominent role in statistical analysis using empirical data. The order statistics are the values from a random sample arranged in an increasing order. For a given random sample, we may interest in the smallest, largest or middle observations, for example: the highest floodwaters, the lowest winter temperature in few past years and the median price of items. We focus to present the cumulative distribution and pdf order statistics for EK-W distribution. Let $X_{1}, X_{2}, \ldots, X_{n}$ be a random sample from a population with distribution function $F(x)$ and pdf $f(x)$. The corresponding order statistics of this sample is $X_{1: n}<X_{2: n}<\cdots<X_{n: n}$. Let $f_{r: n}(x)$ and $F_{r: n}(x)$ denote, respectively, to the pdf and cdf of the r-th order statistics $X_{r: n}(1 \leq r \leq n)$. We have

$$
f_{r: n}(x)=\frac{n !}{(r-1) !(n-r) !} f(x) F^{r-1}(x)[1-F(x)]^{n-r}
$$

that can be rewritten as

$$
f_{r: n}(x)=\frac{n !}{(r-1) !(n-r) !} \sum_{l=0}^{n-r}(-1)^{l}\left(\begin{array}{c}
n-r \\
l
\end{array}\right) F^{l+r-1}(x) f(x)
$$

Also, the cdf is given by

$$
F_{r: n}(x)=\frac{n !}{(r-1) !(n-r) !} \sum_{l=0}^{n-r} \frac{(-1)^{l}}{r+l}\left(\begin{array}{c}
n-r \\
l
\end{array}\right) F^{r+1}(x) .
$$

Considering $f(x)$ and $F(x)$ be the pdf and cdf of the EK-W distribution, we can get, using equation (2) and equation (3) the pdf of the r-th order statistic $X_{r: n}$ as

$$
f_{r: n}(x)=\frac{a b \theta}{B(r, n-r+1)} \sum_{l=0}^{n-r} \sum_{k=0}^{\infty} T_{k}(i, j, l) f_{W}\left(x ; \lambda_{k}, \beta\right)
$$

and its cdf as

$$
F_{r: n}(x)=\frac{1}{B(r, n-r+1)} \sum_{l=0}^{n-r} \sum_{k=0}^{\infty} V_{k}(i, j, l) e^{-k(\lambda x)^{\beta}}
$$

where

$$
\begin{aligned}
T_{k}(i, j, l)= & \sum_{i=0}^{\infty} \sum_{j=0}^{\infty}\left(\begin{array}{c}
n-r \\
l
\end{array}\right) \times \\
& \frac{(-1)^{i+j+k+l} \Gamma[(l+r) \theta] \Gamma[b(i+1)] \Gamma[a(j+1)]}{i ! j ! k ! \Gamma[(l+r) \theta-i] \Gamma[b(i+1)-j] \Gamma[a(j+1)-k](k+1)}
\end{aligned}
$$

and

$$
\begin{aligned}
V_{k}(i, j, l)= & \sum_{i=0}^{\infty} \sum_{j=0}^{\infty} \\
& \frac{(-1)^{i+j+k+l} \Gamma[(l+r) \theta+1] \Gamma[b i+1] \Gamma[a j+1]}{i ! j ! k !(l+r) \Gamma[(l+r) \theta-i+1] \Gamma[b i-j+1] \Gamma[a j-k+1]} .
\end{aligned}
$$

\section{Hazard Function}

Hazard rate function (hf) measures the conditional probability of failure given the system (component) is currently functioning while the pdf (failure density) measures the overall speed of failures. For a continuous distribution with pdf $f(x)$ and reliability function $R(x)$, the hazard function, $h(x)$, is defined by

$$
h(x)=\lim _{\delta X \rightarrow 0} \frac{P(X<x+\delta x \mid X>x)}{\delta x}=\frac{f(x)}{R(x)} .
$$

For the EK-W distribution, the hazard function is given by

$$
h(x)=\frac{\lambda \beta a b \theta(\lambda x)^{\beta-1} e^{-(\lambda x)^{\beta}} u^{a-1}\left[1-u^{a}\right]^{b-1}\left\{1-\left[1-u^{a}\right]^{b}\right\}^{\theta-1}}{1-\left[1-\left(1-u^{a}\right)^{b}\right]^{\theta}}
$$


where

$$
u=u(x)=1-e^{-(\lambda x)^{\beta}} .
$$

We study the asymptotic behaviors and parametric characterizations of the hazard function of the EK-W distribution.

(i) The asymptote of $h(x)$ as $x \rightarrow 0$ : We have

$$
\lim _{x \rightarrow 0} h(x)=\frac{\lim _{x \rightarrow 0} f(x)}{\lim _{x \rightarrow 0} R(x)} .
$$

The numerator of equation (27) is given by equation (11). The asymptote of $R(x)$ as $x$ approaches zero can be obtained as

$$
\lim _{x \rightarrow 0} R(x) \approx 1-b^{\theta}(\lambda x)^{\beta a \theta} .
$$

Substituting the results in equations (11) and (28) in equation (27), we get

$$
h(x) \approx \frac{\lambda \beta a \theta b^{\theta}(\lambda x)^{\beta a \theta-1}}{1-b^{\theta}(\lambda x)^{\beta a \theta}}, \quad \text { as } \quad x \rightarrow 0 .
$$

Therefore, as $x \rightarrow 0$, we have the following cases:

- When $\beta a \theta=1, h(x) \approx \lambda b^{\theta}$,

- When $\beta a \theta<1, h(x) \approx \infty$,

- When $\beta a \theta>1, h(x) \approx 0$.

Comparing these results with the results of equation (13), we can say that $h(0)=f(0)$.

(ii) The asymptote of $h(x)$ as $x \rightarrow \infty$ : We have

$$
h(\infty)=\lim _{x \rightarrow \infty} \frac{f(x)}{1-F(x)}=\lim _{x \rightarrow \infty} \frac{-f^{\prime}(x)}{f(x)}=\lim _{x \rightarrow \infty} g(x) .
$$

where

$$
\begin{aligned}
g(x)= & \lambda \beta(\lambda x)^{\beta-1}-\lambda(\beta-1)(\lambda x)^{-1}-\lambda \beta(a-1)(\lambda x)^{\beta-1} u^{-1} e^{-(\lambda x)^{\beta}} \\
& -\lambda \beta a b(\theta-1)(\lambda x)^{\beta-1} e^{-(\lambda x)^{\beta}} u^{a-1}\left(1-u^{a}\right)^{b-1}\left[1-\left(1-u^{a}\right)^{b}\right]^{-1} \\
& +\lambda \beta a(b-1)(\lambda x)^{\beta-1} e^{-(\lambda x)^{\beta}} u^{a-1}\left(1-u^{a}\right)^{-1}
\end{aligned}
$$

and $u=u(x)=1-e^{-(\lambda x)^{\beta}}$.

A discussion of the limiting of $g(x)$ as $x \rightarrow \infty$ through the following cases, we can see that:

- At $\beta=1$ : We get

$$
\begin{gathered}
g(x)=\lambda-\lambda(a-1) u_{1}^{-1} e^{-\lambda x}+\lambda a(b-1) e^{-\lambda x} u_{1}^{a-1}\left(1-u_{1}^{a}\right)^{-1} \\
-\lambda a b(\theta-1) e^{-\lambda x} u_{1}^{a-1}\left(1-u_{1}^{a}\right)^{b-1}\left[1-\left(1-u_{1}^{a}\right)^{b}\right]^{-1}, \\
u_{1}=u_{1}(x)=1-e^{-\lambda x} \rightarrow 1 \quad \text { as } \quad x \rightarrow \infty .
\end{gathered}
$$

Thus, $g(x) \approx \lambda+\lambda(b-1)=\lambda b$.

- At $\beta>(<) 1$ : We have the expansion

$$
1-u_{1}^{a}=a e^{-(\lambda x)^{\beta}}\left[1+(a-1) e^{-(\lambda x)^{\beta}}-o\left(e^{-(\lambda x)^{\beta}}\right)\right]
$$

Using this approximation when $\beta>1$ and $x \rightarrow \infty$, we can finally see that $g(x) \rightarrow \infty$. Also, it is easy to see that $g(x) \rightarrow 0$ as $x \rightarrow \infty$ when $\beta<1$. From the above discussion, we conclude, as $x \rightarrow \infty$, that

- When $\beta<1, h(x) \approx 0$,

- When $\beta=1, h(x) \approx \lambda b$, 
- When $\beta>1, h(x) \approx \infty$.

The above results can be summarized in the following theorem.

Theorem 3 The hazard rate function of the $E K-W(\lambda, \beta, a, b, \theta)$ distribution has the following asymptotes:

$$
\lim _{x \rightarrow 0} h(x)= \begin{cases}0, & \beta a \theta>1 \\ \lambda b^{\theta}, & \beta a \theta=1 \\ \infty, & \beta a \theta<1\end{cases}
$$

and

$$
\lim _{x \rightarrow \infty} h(x)= \begin{cases}0, & \beta<1 \\ \lambda b, & \beta=1 \\ \infty, & \beta>1 .\end{cases}
$$

Furthermore, parametric characterizations for $h(x)$ of the EK-W distribution can be adopted in the following theorem.

Theorem 4 The hazard rate function, $h(x)$ of the $E K-W(\lambda, \beta, a, b, \theta)$ distribution possesses the following behaviors: ( $i)$ $h(x)$ is constant $(=\lambda b)$ when $\beta=a=\theta=1$. (ii) $h(x)$ is increasing (decreasing) when $\beta \geq 1(\beta \leq 1)$ and $\beta a \theta \geq 1(\beta a \theta \leq 1)$. (iii) $h(x)$ has bathtub-shape curve when $\beta>1$ and $\beta a \theta<1$. (iv) $h(x)$ is unimodal when $\beta<1$ and $\beta a \theta>1$.

Proof. The proof is straightforward from the results of Theorem 3.

Figures 5 and 6 display some plots for $h(x)$ to show and to support these results. Figure 5 displays the monotonic behavior of the hazard function of EK-W at $\beta=1, \beta a \theta=1$. In this figure, the case of constant hf, we considered $\lambda=2$ and $b=5$. Figure 6 displays the monotonic and non-monotonic behaviors of the hazard function (at $\beta \neq 1, \beta a \theta \neq 1$ ) for selected values of the parameters $\beta, a$ and $\theta$.

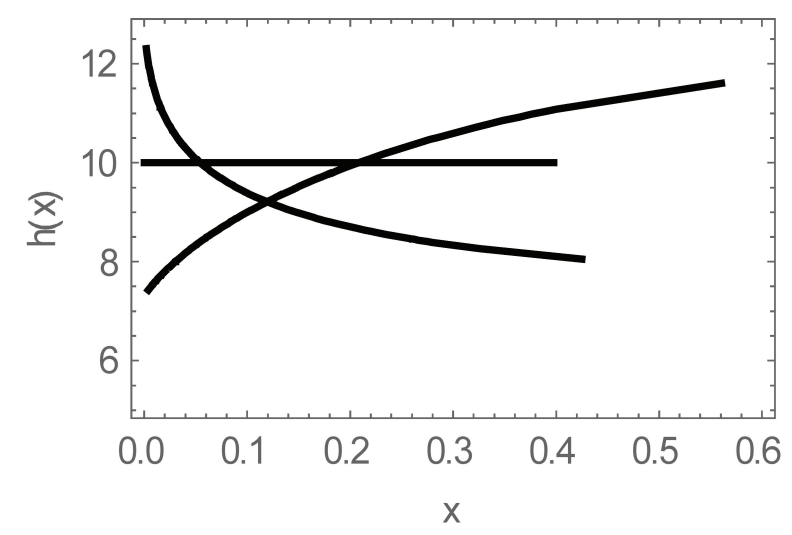

Figure 5. Constant, increasing and decreasing hfs, $\beta=1, \beta a \theta=1$.

\section{Maximum Likelihood Estimation}

The maximum likelihood as a method of estimation for parameters is the most commonly used in the literature. In this study, we discuss the maximum likelihood, ML, estimation for the parameters of the EK-W distribution for complete samples. For a random samples, $x_{1}, x_{2}, x_{3}, \ldots, x_{n}$ of size $n$ from the $E K-W(\lambda, \beta, a, b, \theta)$, the log-likelihood function, $l(\lambda, \beta, a, b, \theta)$, given this sample is given by

$$
l(\lambda, \beta, a, b, \theta)=n \beta \ln \lambda+n \ln \beta+n \ln a+n \ln b+n \ln \theta-\varphi(\lambda, \beta, a, b, \theta)
$$

where

$$
\begin{aligned}
\varphi(\lambda, \beta, a, b, \theta) & =\sum_{i=1}^{n}\left(\lambda x_{i}\right)^{\beta}-(\beta-1) \sum_{i=1}^{n} \ln x_{i}-(a-1) \sum_{i=1}^{n} \ln u_{i} \\
& -(b-1) \sum_{i=1}^{n} \ln \left(1-u_{i}^{a}\right)-(\theta-1) \sum_{i=1}^{n} \ln \left[1-\left(1-u_{i}^{a}\right)^{b}\right]
\end{aligned}
$$




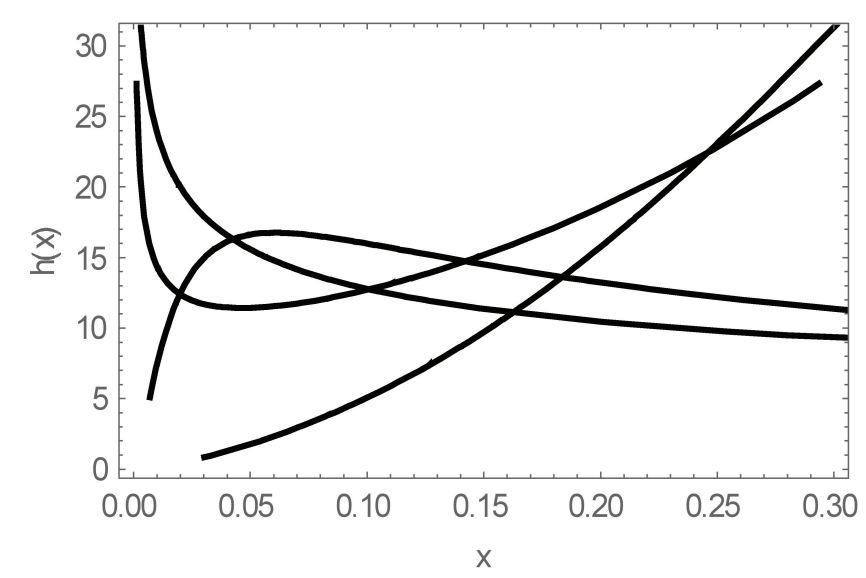

Figure 6. Different behaviors for the hazard function, $\beta \neq 1, \beta a \theta \neq 1$.

$$
u_{i}=u_{i}\left(x_{i}, \lambda, \beta\right)=1-e^{-\left(\lambda x_{i}\right)^{\beta}} .
$$

The ML estimating equations are obtained as follows:

$$
\begin{aligned}
& n \beta-\beta \sum_{i=1}^{n}\left(\lambda x_{i}\right)^{\beta}+\beta(a-1) \sum_{i=1}^{n} V_{i}-\beta a(b-1) \sum_{i=1}^{n} V_{i} u_{i}^{a}\left(1-u_{i}^{a}\right)^{-1} \\
& +\beta a b(\theta-1) \sum_{i=1}^{n} V_{i} u_{i}^{a}\left(1-u_{i}^{a}\right)^{b-1}\left[1-\left(1-u_{i}^{a}\right)^{b}\right]^{-1}=0, \\
& \frac{n}{\beta}+n \ln \lambda-\sum_{i=1}^{n}\left(\lambda x_{i}\right)^{\beta} \ln \left(\lambda x_{i}\right)+\sum_{i=1}^{n} \ln x_{i}+(a-1) \sum_{i=1}^{n} V_{i} \ln \left(\lambda x_{i}\right) \\
& -a(b-1) \sum_{i=1}^{n} V_{i} u_{i}^{a}\left(1-u_{i}^{a}\right)^{-1} \ln \left(\lambda x_{i}\right) \\
& +a b(\theta-1) \sum_{i=1}^{n} V_{i} u_{i}^{a}\left(1-u_{i}^{a}\right)^{b-1}\left[1-\left(1-u_{i}^{a}\right)^{b}\right]^{-1} \ln \left(\lambda x_{i}\right)=0, \\
& \frac{n}{a}+\sum_{i=1}^{n} \ln u_{i}-(b-1) \sum_{i=1}^{n} u_{i}^{a}\left(1-u_{i}^{a}\right)^{-1} \ln u_{i} \\
& +b(\theta-1) \sum_{i=1}^{n} u_{i}^{a}\left(1-u_{i}^{a}\right)^{b-1}\left[1-\left(1-u_{i}^{a}\right)^{b}\right]^{-1} \ln u_{i}=0 \\
& \frac{n}{b}+\sum_{i=1}^{n} \ln \left(1-u_{i}^{a}\right) \\
& -(\theta-1) \sum_{i=1}^{n}\left(1-u_{i}^{a}\right)^{b}\left[1-\left(1-u_{i}^{a}\right)^{b}\right]^{-1} \ln \left(1-u_{i}^{a}\right)=0 \\
& \frac{n}{\theta}+\sum_{i=1}^{n} \ln \left[1-\left(1-u_{i}^{a}\right)^{b}\right]=0
\end{aligned}
$$

where $V_{i}=\left(\lambda x_{i}\right)^{\beta} u_{i}^{-1}\left(1-u_{i}\right)$.

Solving this system of equations (30)-(34) simultaneously gives the ML estimates of the five parameters. This system of nonlinear estimating equations cannot be solved analytically. Numerical technique such as Newton-Raphson iterative method should be adopted to obtain the ML estimates, $\hat{\lambda}, \hat{\beta}, \hat{a}, \hat{b}, \hat{\theta}$ of the different parametrs. For this purpose, the $\mathrm{R}$ project or Matlab software is preferable to use. 


\section{Applications to Real Data}

In this section, we present some applications of the EK-W distribution using different data sets to demonstrate the flexibility of the distribution to model these real data. The data sets include a skewed and heavy-tailed data. The data are fitted to the EK-W distribution and other Kumaraswamy distributions family, the K-W and EK-E distributions, as well as the EW distribution. The first data set (it is not cited here to save space) represents the sum of skin folds in 202 athletes collected at the Australian Institute of Sports and previously reported by Weisberg (2005). The second data set taken from Murthy et al. (2004, page 180) represents the failure times of 50 components (per 1000 hours). The third data set is from Aarset (1987) and it represents the time to failure of 50 industrial devices put on life test at time zero. The summary statistics from the three data sets are shown in Table 2. The notations used in Table 2: $\bar{x}, \mathrm{SD}, \gamma_{1}$ and $\gamma_{2}$ are the sample mean, standard deviation, skewness and kurtosis, respectively.

Data set 2:

$0.036,0.058,0.061,0.074,0.078,0.086,0.102,0.103,0.114,0.116,0.148,0.183,0.192,0.254,0.262,0.379,0.381$, $0.538,0.570,0.574,0.590,0.618,0.645,0.961,1.228,1.600,2.006,2.054,2.804,3.058,3.076,3.147,3.625,3.704$, $3.931,4.037,4.393,4.534,4.893,6.274,6.816,7.896,7.904,8.022,9.337,10.940,11.020,13.880,14.730,15.080$.

Data set 3:

$0.1,0.2,1,1,1,1,1,2,3,6,7,11,12,18,18,18,18,18,21,32,36,40,45,46,47,50,55,60,63,63,67,67,67,67,72,75$, $79,82,82,83,84,84,84,85,85,85,85,85,86,86$.

We obtain the ML estimates of the parameters of the distribution under consideration by employing and solving the system of equations 30-34, numerically, as well as calculate the log-likelihood value $(\hat{\ell})$, the Akaike Information Criterion (AIC), the Kolmogorov-Smirnov (K-S) test statistic, and the p-value for the K-S statistic for the fitted distributions to the data sets. We report the results in Tables 3- 8. Figures 7 and 8 display the histograms and the fitted density functions for the first and second data sets, that support the results in Tables 3 and 5. Figures 9-12 display the Q-Q plots for Aarset data quantiles and fitted quantiles for the different models. From Tables 3, 5 and 7, the goodness of fit statistics indicate that the EK-W distribution provides the best fit among the distributions K-W, EK-E and EW, since it has the smallest AIC and $\mathrm{K}-\mathrm{S}$ statistics, and the largest log-Likelihood value and p-value of K-S test statistic (at 0.05 significance level).

In view of Theorem 4 and Theorem 1 and in terms of the parameters estimates of EK-W distribution for the different data sets, we have the following notes:

- The data set 1 has increasing hazard rate function since $\beta>1$, and $\beta a \theta>1$, as well as the pdf is unimodal.

- The hazard rate function of data set 2 is decreasing since $\beta<1$, and $\beta a \theta<1$, as well as the pdf is decreasing function.

- The hazard rate function of Aarset data set 3 has bathtub shape curve since $\beta>1$, and $\beta a \theta<1$, as well as the pdf is decreasing function.

Finally, in the light of the above results, the exponentiated Kumaraswamy-Weibull distribution provides a good fit for these real data sets as compared to its sub models.

Table 2. Summary statistcs from the data sets.

\begin{tabular}{l|c|c|c|c}
\hline Data sets & $\bar{x}$ & SD & $\gamma_{1}$ & $\gamma_{2}$ \\
\hline Skin folds & 69.027 & 32.560 & 1.175 & 3.366 \\
Failure times of components & 3.343 & 4.181 & 1.417 & 4.085 \\
Life tmes of industrial devices & 46.686 & 32.835 & -0.138 & 1.414 \\
\hline
\end{tabular}

Table 3. Information criteria for fitting the sum of skin folds data.

\begin{tabular}{l|c|c|c|c}
\hline Model & $-\hat{\ell}$ & $A I C$ & $k-S$ & $p-$ value \\
\hline$E K-W$ & 954.768 & 1919.54 & 0.0692 & 0.2751 \\
$K-W$ & 958.927 & 1925.85 & 0.0774 & 0.1685 \\
$E K-E$ & 958.083 & 1924.17 & 0.0773 & 0.1694 \\
$E W$ & 957.756 & 1921.51 & 0.0920 & 0.0615 \\
\hline
\end{tabular}


Table 4. Parameter estimates for the sum of skin folds data.

\begin{tabular}{l|c|c|c|c|c}
\hline Model & $\hat{\lambda}$ & $\hat{\beta}$ & $\hat{a}$ & $\hat{b}$ & $\hat{\theta}$ \\
\hline$E K-W$ & 0.0843 & 1.0018 & 7.2135 & 0.4343 & 2.5291 \\
$K-W$ & 0.0349 & 1.0206 & 6.8832 & 1.1095 & 1 \\
$E K-E$ & 0.0049 & 1 & 0.9846 & 7.8775 & 8.0012 \\
$E W$ & 0.0472 & 0.9128 & 1 & 1 & 9.5333 \\
\hline
\end{tabular}

Table 5. Information criteria for fitting the failure times of 50 components.

\begin{tabular}{l|c|c|c|c}
\hline Model & $-\hat{\ell}$ & AIC & $k-S$ & $p-$ value \\
\hline$E K-W$ & 100.809 & 210.618 & 0.1141 & 0.4974 \\
$K-W$ & 104.061 & 216.122 & 0.1891 & 0.0487 \\
$E K-E$ & 102.156 & 212.313 & 0.1341 & 0.3017 \\
$E W$ & 102.372 & 211.745 & 0.1429 & 0.2351 \\
\hline
\end{tabular}

Table 6. Parameter estimates for the failure times of 50 components.

\begin{tabular}{l|c|c|c|c|c}
\hline Model & $\hat{\lambda}$ & $\hat{\beta}$ & $\hat{a}$ & $\hat{b}$ & $\hat{\theta}$ \\
\hline$E K-W$ & 2.3142 & 0.9781 & 2.7199 & 0.0644 & 0.3198 \\
$K-W$ & 1.2615 & 0.9440 & 0.4141 & 0.2134 & 1 \\
$E K-E$ & 0.4910 & 1 & 1.7365 & 0.3440 & 0.3402 \\
$E W$ & 0.1939 & 0.9997 & 1 & 1 & 0.5365 \\
\hline
\end{tabular}

Table 7. Information criteria for fitting the failure times of 50 devices.

\begin{tabular}{l|c|c|c|c}
\hline Model & $-\hat{\ell}$ & AIC & $k-S$ & $p-$ value \\
\hline$E K-W$ & 235.557 & 481.115 & 0.1661 & 0.1127 \\
$K-W$ & 237.570 & 483.141 & 0.1957 & 0.0375 \\
$E K-E$ & 239.999 & 488.000 & 0.2010 & 0.0116 \\
$E W$ & 239.791 & 485.583 & 0.2228 & 0.0302 \\
\hline
\end{tabular}

Table 8. Parameter estimates for the failure times of 50 devices.

\begin{tabular}{l|c|c|c|c|c}
\hline Model & $\hat{\lambda}$ & $\hat{\beta}$ & $\hat{a}$ & $\hat{b}$ & $\hat{\theta}$ \\
\hline$E K-W$ & 0.0629 & 1.0925 & 0.0332 & 0.3729 & 5.5715 \\
$K-W$ & 0.0598 & 1.1278 & 0.5920 & 0.2845 & 1 \\
$E K-E$ & 0.0184 & 1 & 0.8817 & 1.0000 & 0.8783 \\
$E W$ & 0.0195 & 1.0626 & 1 & 1 & 0.8221 \\
\hline
\end{tabular}

\section{Conclusion}

We proposed a new five-parameter distribution, named the exponentiated Kumaraswamy-Weibull -distribution, which extends the Kumaraswamy-Weibull distribution and other important well-known distributions such as EW, ERay, EK-E and Weibull. Various properties for this distribution are studied including the asymptotes and parametric characterizations for the probability density and hazard functions. Approximate form of the mode is derived. We obtain the moments, moment generating function, Bonferroni and Lorenz curves, entropy, mean deviations and the probability density and cumulative distribution functions of order statistics. The estimation of the parameters has been discussed using the ML method. Analysis for three real data sets reveals that the EK-W distribution is quite flexible and effective model in fitting real world data compared with its sub models. 


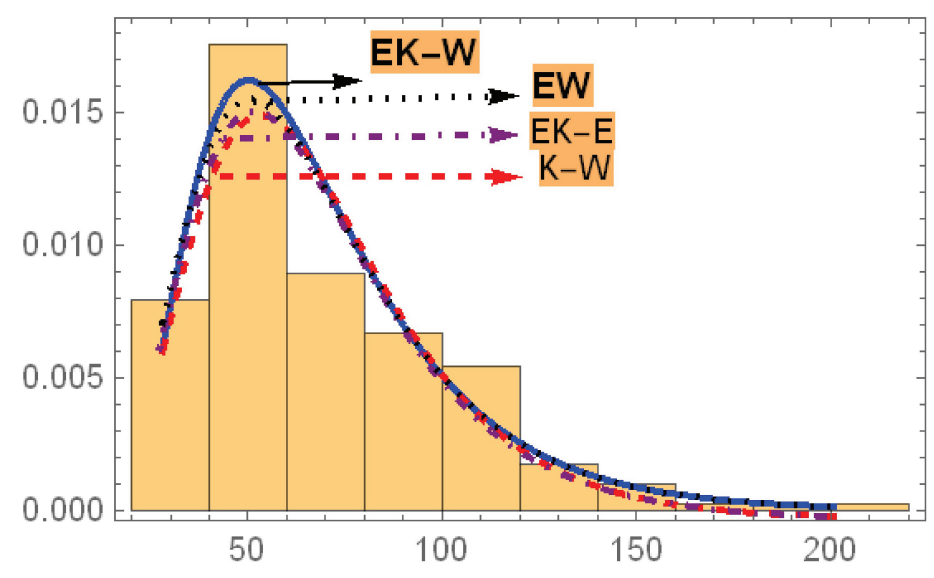

Figure 7. Estimated pdfs of the different models for data set 1.

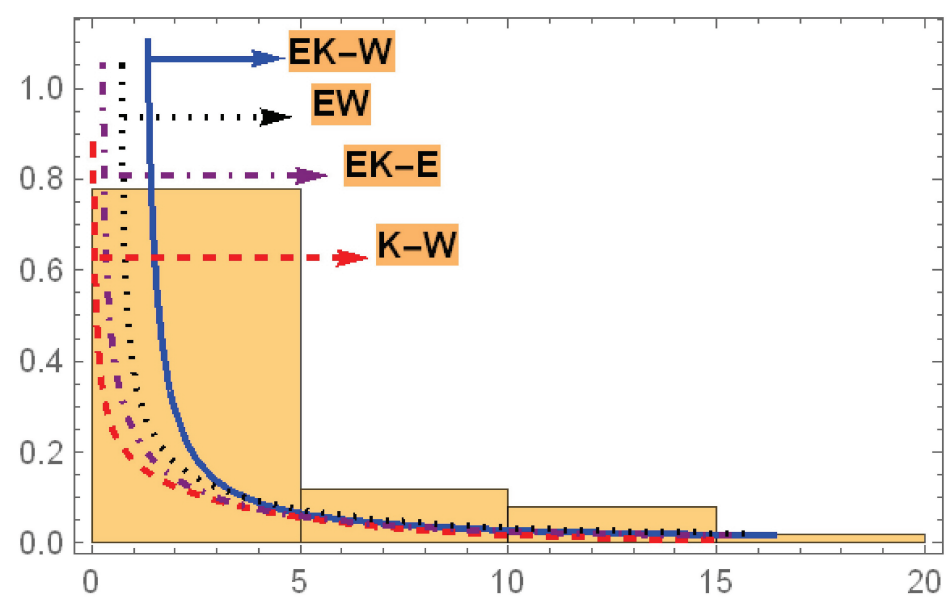

Figure 8. Estimated pdfs of the different models for data set 2. 


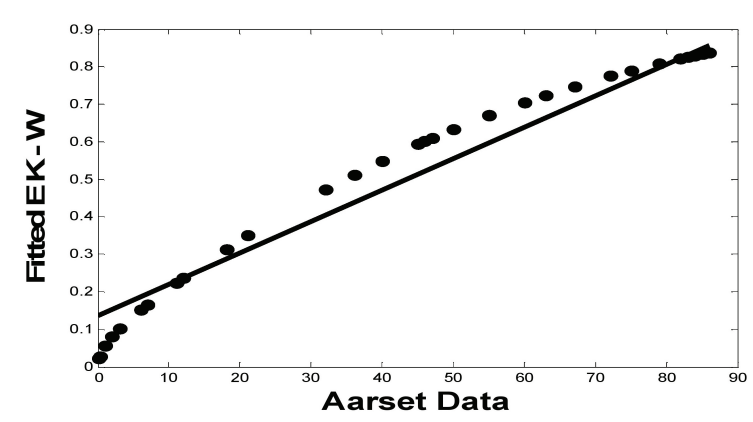

Figure 9. Q-Q plot of fitted EK-W to Aarset data.

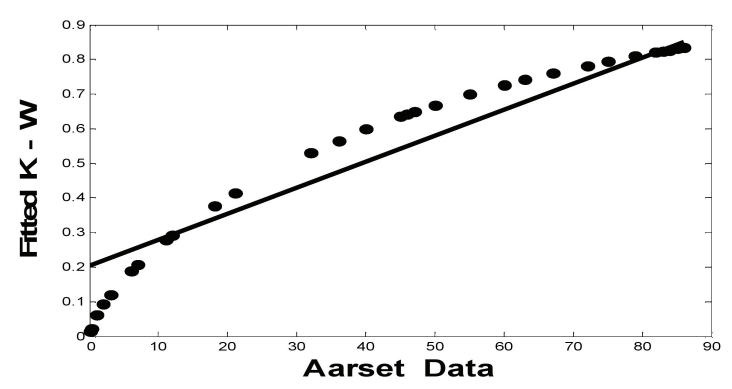

Figure 10. Q-Q plot of fitted K-W to Aarset data.

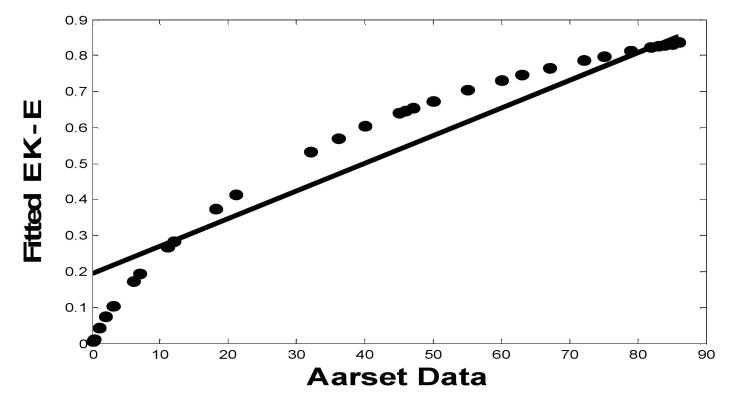

Figure 11. Q-Q plot of fitted EK-E to Aarset data.

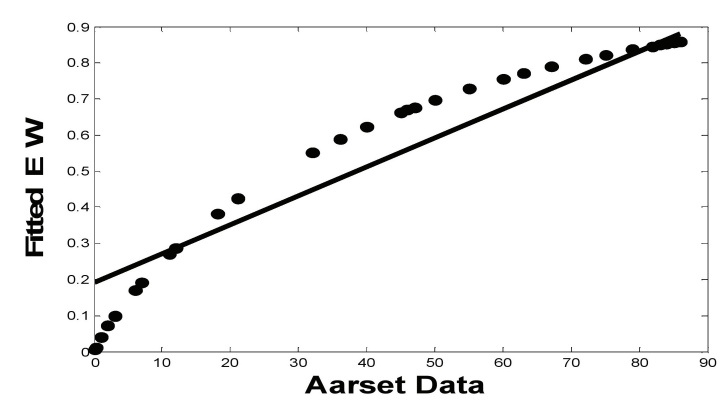

Figure 12. Q-Q plot of fitted EW to Aarset data. 


\section{References}

Aarset, M. V. (1987). How to identify bathtub hazard rate. IEEE Transaction in reliability, 36, 106-108. https://doi: 0018-9529/87/0400-0106\$01.00.

Cordeiro, G. M., \& de Castro, M. A. (2011). A new family of generalized distributions. Journal of Statistical Computation and Simulation, 81, 883-898. https://doi: 10.1080/0094965YY.

Cordeiro, G. M., Ortega, E. M. M., \& Nadarajah, S. (2010). The Kumaraswamy generalized gamma distribution with application in survival analysis. Statistical Methodology, 8, 411-433. https://doi: 10.1016/j.stamet. 2011.04.001.

de Pascoa, M. A. R., Ortega, E. M. M., \& Cordeiro, G. M. (2011). The Kumaraswamy Weibull distribution with application to failure data. Journal of Franklin Institute, 347, 1399-1429. https://doi: 10.1016/j.jfranlin.2010.06.010.

Galton, F. (1983). Enquiries into Human Faculty and its Development. London: Macmillan and Company.

Gomes, A. E., da Silva, C. Q., Cordeiro, G. M., \& Ortega, E. M. M. (2014). A new lifetime model: The Kumaraswamy generalized Rayleigh distribution. Journal of Statistical Computation and Simulation, 84, 290-309. https://doi: 10.1080/00949655.2012.706813.

Huang, S., \& Oluyede, B.O. (2014). Exponentiated Kumaraswamy-dagum distribution with applications to income and lifetime data. Journal of Statistical Distributions and Applications, 1, 1-20. https://doi: 10.1186/2195-5832-1-8.

Jones, M. C. (2009). Kumaraswamy's distribution: A beta-type distribution with some tractability advantages. Statistical Methodology, 6, 70-91. https://doi: 10.1016/j.stamet.2008.04.001.

Kumaraswamy, P. (1980). Generalized probability density- function for double-bounded random processes. Journal of Hydrology, 46, 79-88. https://doi: 10.1016/0022-1694(80)90036-0.

Moors, J. J. (1988). A quantile alternative for kurtosis. Statistician, 37, 25-32. https://doi: 10.2307/2348376.

Murthy, D. P., Xie, M., \& Jiang, R. (2004). Weibull Models. New York: John Wiley \& Sons.

Rodrigues, J.A., \& Silva, A. P. C. (2015). The exponentiated Kumaraswamy-exponential distribution. British Journal of Applied Science and Technology, 10, 1-12. https://doi: 10.9734/BJAST/2015/16935.

Paranaiba, P. F., Ortega, E. M. M., Cordeiro, G. M., \& Pascoa, M. A. D. (2012). The Kumaraswamy Burr XII distribution: Theory and practice. Journal of Statistical Computation and Simulation, 82(3), 1-27. https://doi: 10.1080/00949655.2012.683003.

Weisberg, S. (2005). Applied Linear Regression. New York, NY: John Wiley \& Sons.

\section{Copyrights}

Copyright for this article is retained by the author(s), with first publication rights granted to the journal.

This is an open-access article distributed under the terms and conditions of the Creative Commons Attribution license (http://creativecommons.org/licenses/by/4.0/). 